\title{
Presentation of new onset anosmia during the COVID-19 pandemic ${ }^{*}$
}

\author{
Claire Hopkins ${ }^{1,2}$, Pavol Surda', B. Nirmal Kumar ${ }^{3}$ \\ Guy's and St Thomas'Hospitals, London, United Kingdom \\ King's College, London, United Kingdom \\ Edge Hill University Medical School, Orsmkirk, United Kingdom
}

Rhinology 58: 3, 295 - 298, 2020

https://doi.org/10.4193/Rhin20.116

*Received for publication:

March 28, 2020

Accepted: April 7, 2020

\begin{abstract}
Introduction: Anosmia has not been formally recognised as a symptom of COVID-19 infection. Growing anecdotal evidence suggests increasing incidence of cases of anosmia during the current pandemic, suggesting that COVID-19 may cause olfactory dysfunction. The objective was to characterise patients reporting new onset anosmia during the COVID-19 pandemic
\end{abstract}

Methodology: Design: Survey of 2428 patients reporting new onset anosmia during the COVID-19 pandemic.

Setting: Volunteer sample of patients seeking medical advice of recent onset self-diagnosed loss of sense of smell

Results: 2428 surveys were completed within 7 days; $64 \%$ respondents were under 40 . The majority of respondents reported onset of their anosmia in the last week. Of the cohort, $17 \%$ did not report any other symptom thought to be associated with COVID-19. In patients who reported other symptoms, 51\% reported either cough or fever and therefore met current guidelines for self-isolation

Conclusions: Anosmia is reported in conjunction with well-reported symptoms of coronas virus, but 1 in 6 patients with recent onset anosmia report this as an isolated symptom. This might help identify otherwise asymptomatic carriers of disease and trigger targeted testing. Further study with COVID-19 testing is required to identify the proportion of patients in whom new onset anosmia can be attributed to COVID-19

Key words: SARS-CoV-2, COVID-19, anosmia, epidemiology

\section{Introduction}

Post-Viral anosmia is one of the leading causes of loss of sense of smell in adults accounting for up to $40 \%$ cases of anosmia(1). Viruses that give rise to the common cold are well known to cause post-infectious loss, and over 200 different viruses known to cause upper respiratory tract infections. Previously described coronaviruses are thought to account for $10-15 \%$ cases $^{(2)}$. Human strains of corona virus have been demonstrated to invade the central nervous system through the neuroepithelium and propagate from within the olfactory bulb(3).

It is therefore perhaps no surprise that the novel COVID-19 virus could also cause anosmia in infected patients. There is emerging non-peer reviewed evidence that significant numbers of pa- tients with proven COVID-19 infection have developed anosmia/ hyposmia. In Germany, one study reported in the press, stated that more than 2 in 3 confirmed reported loss of smell and taste, although they did not enquire about timing of onset ${ }^{(4)}$. In addition, there have been a rapidly growing number of anecdotal reports of a significant increase in the number of patients presenting with anosmia who otherwise have mild symptoms or in the absence of other symptoms - this has been widely shared on medical discussion boards by surgeons from all regions managing a high incidence of COVID-19 cases. In a study of 3191 COVID-19+ patients who were self-isolating at home with mild disease, $15.3 \%$ expressed smell or taste loss ${ }^{(5)}$.

The authors noted a sudden increase in young, health and 
otherwise asymptomatic patients presenting with recent onset of anosmia in the past weeks, prompting the release of a pressrelease aimed to highlight the potential link to fellow ENT surgeons and encourage use of personal protective equipment (PPE) in such cases. Following the release of the statement there has been considerable media attention as both, celebrities, politicians and many others have shared their recent onset of anosmia on social media - both in confirmed and unconfirmed cases. The first author received a deluge of emails from colleagues and patients reporting recent onset anosmia, seeking advice and volunteering help.

\section{Methodology}

\section{Survey}

A simple survey to audit the onset of anosmia and associated symptoms was designed and sent to patients making contact by email for advice on their new onset anosmia, along with an advice sheet. The survey was conducted anonymously, and no reward was offered for completion. Many patients confirmed that they had completed the survey and also reported that they had shared the survey with multiple immediate family members, close friends and colleagues who were similarly afflicted. Within 24 hours of sending approximately 300 email replies, 710 respondents had completed the survey. At 7 days, 2428 responses had been received, after sending approximately 400 replies to patient queries, suggesting wider dissemination. The database was checked for duplicate replies, but all completed surveys appeared unique.

\section{Results}

$64 \%$ of 2428 respondents were aged under 40 , (Figure 1), and the median was 30 - 39years. $73 \%$ were female.

The majority of respondents (61\%) report onset of their anosmia less than 1 week before completing the survey. The timing of onset mirrors the rising incidence of COVID-19 in the UK (Figure 2). Of the cohort, 16\% did not report any other symptom thought to be associated with COVID-19. In patients who reported other symptoms, the most common were cough (Figure 3 ), and 51\% reporting either cough or fever, and therefore met current UK guidelines for social isolation or likely diagnosis of COVID-19 infection. Unfortunately, the timing of onset in relation to other symptoms was not included in the questionnaire at initiation and was added shortly after data collection started, but of the 1325 completed responses where patients experienced other symptoms, $13 \%$ reported anosmia before their onset, $38.4 \%$ at the same time, and in $48.6 \%$ the onset of anosmia came after other symptoms.

$74.4 \%$ reported complete loss of sense of smell, a further $17.3 \%$ reported a very severe loss. $90 \%$ reported that their sense of

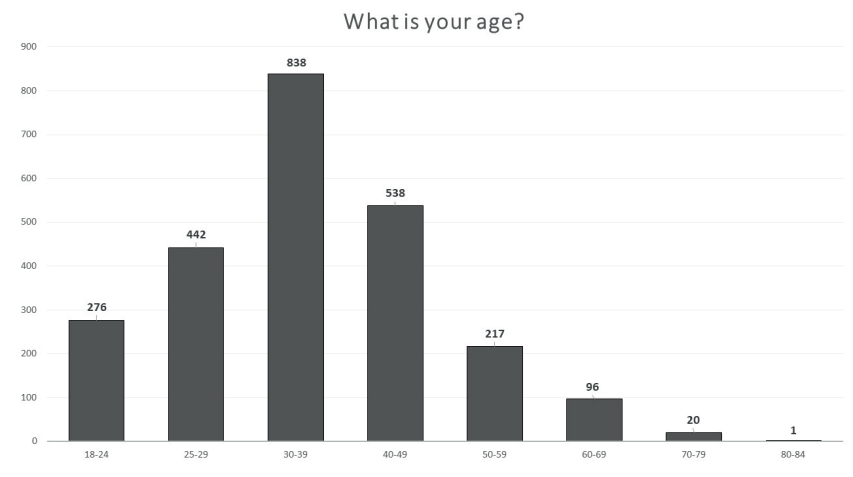

Figure 1. Age distribution of respondents.

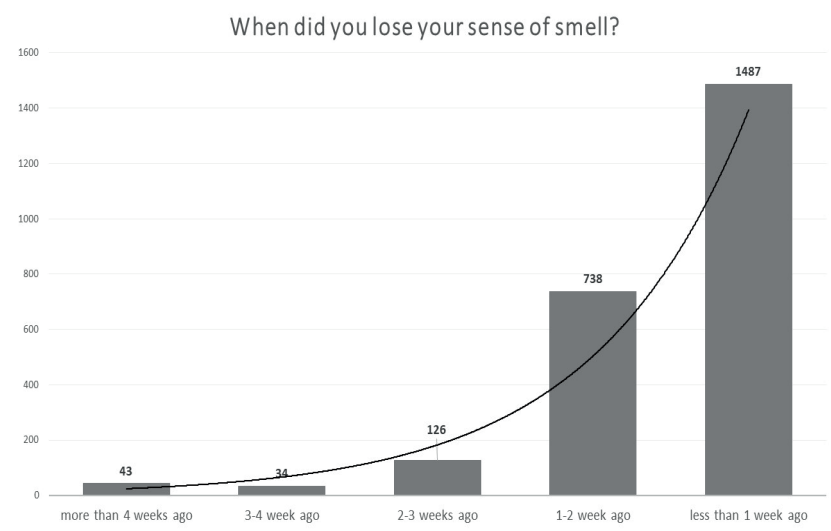

Figure 2. Timing of onset of anosmia

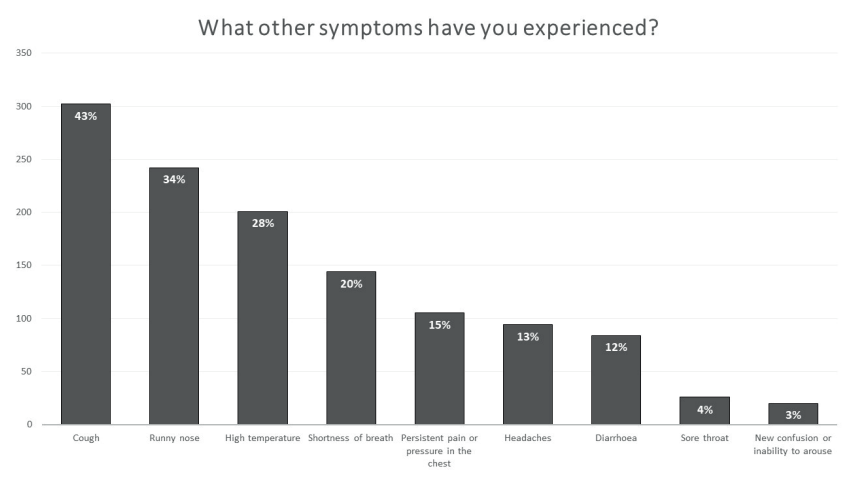

Figure 3. Prevalence of associated symptoms.

taste was reduced, but $61 \%$ of the whole group reported that they could still differentiate between sweet, salty, sour and bitter tastes, suggesting the predominant issue is reduction in sense of smell rather than hypogeusia, although true taste disturbance should be considered. In free text, some patients also reported altered taste or a metallic taste, although we are not able to further determine if this reflects true dysgeusia. 35\% were already noticing improvement at the time of completing the survey.

Only 80 patients had been tested for COVID-19, of which $74 \%$ have tested positive. $14.9 \%$ of patients had not self-isolated. $10.1 \%$ had a known contact who had tested positive for COVID-19. 


\section{Discussion}

Severe olfactory loss is estimated to have an estimated prevalence of around $5 \%$ in population studies ${ }^{(6)}$. Risk factors are increasing age and male gender; with significant increases in the incidence of olfactory function not seen in studies before the age of 45-49 in males, and older in females ${ }^{(7,8)}$.

Our cohort are younger than typically reported as having higher rates of olfactory loss. Only a small proportion in our study were over 70 , but this may reflect selection bias in the type of person will complete an online survey, use of email as the point of contact, or the increased prevalence of pre-existing anosmia in this age group. It might be that in older age groups, who are reported to present with more severe symptoms, anosmia is neglected as the focus is on respiratory care.

An apparent female predominance needs further study, as it may simply reflect gender differences in completing and sharing the survey.

Post-viral anosmia is a common cause of olfactory loss, and transient loss is likely under-reported such that the true incidence is unknown. There is a chance the apparent increase in incidence could merely reflect the attention COVID19 has attracted in the media, and that such cases may be caused by unrelated rhinovirus and coronavirus strains, that would have previously gone unreported. However, there is growing anecdotal evidence to support a link with COVID-19 infection. Internet searches for loss of sense of smell and visits to patient support groups have risen significantly in recent weeks, preceding any reports in social media or the press of a possible link between anosmia and COVID-19 infection ${ }^{(9)}$. Notably, increases in searches occurred in Italy before similar increases in UK users. There are a small number of case-series emerging in the peer reviewed literature; Giacomelli et al interviewed 59 hospitalised COVID-19 patients and found $34 \%$ reported smell or taste disturbance ${ }^{(10)}$. Although the majority of our cohort do meet current criteria for testing in the UK, as it is only being undertaken in hospitalised patients, of those tested in our survey, $74 \%$ were found to be positive, While we do not know the means of testing, but in most cases RT-PCR analysis of nasal swabs has been used, which have an estimated sensitivity of $72 \%{ }^{(11)}$. Many patients also reported symptoms described in COVID-19 infection, with more than half reporting a new onset cough or fever. However, our assumption that our cohort reflects patients with COVID-19 cannot be proven without test results.

Of particular interest are the 1 in 6 patients who have developed anosmia in the absence of any other symptoms - previously these patients would not be suspected to have COVID19 and may be some of the hitherto hidden carriers that have facilitated the rapid spread of infection. $18 \%$ of our cohort had not been self-isolating, in keeping with current guidelines. New onset anosmia (in the absence of a recent head injury or severe nasal obstruction) could therefore potentially be used a screening tool to help identify otherwise asymptomatic patients who continue to act as vectors, and who then could be better instructed on self-isolation or submitted for testing. Overall, nearly 1 in 4 respondents reported anosmia as an isolated symptom, or as the first presenting symptom.

Perhaps more importantly it could be used as a tool to target testing of frontline healthcare workers, to prevent nosocomial spread of disease. Colleagues in Italy have anecdotally reported very high rates of anosmia in front-line health care worked (personal communication), and an immediate colleague of the first author has tested positive having reported isolated anosmia 72 hours ago. He has subsequently self-isolated; detection has reduced risk to medical staff and patients who might otherwise have been exposed. In addition, anomsia must be considered an important trigger for healthcare personnel to employ full PPE and avoid nasal endoscopy in order to help counter the higher rates of infection found amongst ENT surgeons compared to other healthcare workers.

The major limitation of this study is the lack of confirmed COVID-19 status, as discussed above. However, as testing is currently restricted to only those hospitalised in the UK, our cohort are largely ineligible for testing. While the survey was initially sent to patients making contact for advice on new onset anosmia, the survey has been widely disseminated beyond this initial cohort. $98 \%$ reported onset of anosmia within the last 4 weeks, but we have been unable to verify this with objective testing or review of medical records. We cannot estimate the overall prevalence of anosmia in those infected with COVID-19, however unless widespread testing in the community is undertaken, any study in those hospitalised is also prone to selection bias in evaluating a more severely affected, and likely older cohort. The benefit of this study is that it may offer a picture of patients who would likely be missed by current diagnostic criteria, selected populations with severe disease requiring hospitalisation or targeted testing regimens.

A comprehensive review of the management of post-viral anosmia is beyond the scope of this short report. High rates of spontaneous recovery have been reported, ranging from $32 \%{ }^{(12)}$ to $67 \%{ }^{(13)}$ in the absence of any active treatment. Oral steroids, but not topical steroids have been shown to improve olfactory function in a group including post-viral anosmia ${ }^{(14)}$. However, current WHO guidance ${ }^{(15)}$ is to avoid the use of systemic steroids in cases with or at risk of severe acute respiratory COVID-19 as systematic review suggests possible harm including delayed viral clearance. Of patients who develop severe respiratory disease, the median time from onset of symptoms ranged from 
8 - 12 days in reports; therefore, oral steroids should npt be prescribed in the first 2 weeks of suspected COVID-19 post-viral anosmia, and should be considered only in cases where anosmia persists beyond at least 14 days and after careful discussion of likely risks and benefits. Alpha lipoic acid ${ }^{(16)}$ has been shown to improve objective tests of olfactory function in a small uncontrolled study. Omega-3 supplementation was found to protective against olfactory loss during the recovery period after skull base surgery, and therefore may have potential in recovery after post-viral loss ${ }^{(17)}$, although this has not been formally tested. A meta-analysis of 13 studies of olfactory training suggest small to moderate benefit ${ }^{(18)}$. Intranasal Vitamin A added to olfactory training achieved greater rates of improvement compared with olfactory training alone ${ }^{(18)}$. However, almost all studies reported have very small series, most lack a blinded, randomised, control arm and rates of improvement are usually no greater than reported rates of spontaneous improvement.

\section{Conclusion}

While formal research is needed to establish the incidence of anosmia in COVID-19+ patients, along with more widespread testing of patients with new onset anosmia, this is one of the first reports to document the clinical presentation of patients developing anosmia during the pandemic prior to formal testing for COVID-19, and may be used to help inform the design of future studies. Such studies may include objective testing of both smell and taste to better define the subjective reports described herein.

\section{Authorship contribution}

$\mathrm{CH}$ : Study design, data collection and interpretation, drafting of manuscript; PS: data collection and interpretation, drafting of manuscript; NK: study design, review of final manuscript.

\section{Conflict of interest}

The authors have no conflict of interest to declare.

\section{References}

1. Zhu N, Zhang D, Wang W et al. A Nove Welge-Lussen A, Wolfensberger $M$ Olfactory disorders following upper respiratory tract infections. Adv Otorhinolaryngol. 2006;63:125-132

2. Eccles R. Understanding the symptoms of the common cold and influenza. Lancet Infect Dis. 2005:5(11):718-725.

3. Dube M, Le Coupanec A, Wong AHM, Rini JM, Desforges M, Talbot PJ. Axonal Transport Enables Neuron-to-Neuron Propagation of Human Coronavirus OC43. J Virol 2018;92(17).

4. https://www.faz.net/aktuell/gesellschaft/ gesundheit/coronavirus/neue-coronasymptome-entdeckt-virologe-hendrikstreeck-zum-virus-16681450.html

5. ht tps://news.joins.com/ article/23738003?cloc=joongang-mhomegroup6\&fbclid=IwAR33__i-aKtLN2MzC s5AE-

6. Bramerson A, Johansson L, Ek L, Nordin S Bende M. Prevalence of olfactory dysfunction: the skovde population-based study. Laryngoscope. 2004;114(4):733-737.

7. Stogbauer J, Wirkner K, Engel C, et al. Prevalence and risk factors of smell dysfunction - a comparison between five German population-based studies. Rhinology. 2020 Apr 1;58(2):184-191.

8. Wang $X$, Zhang $C$, Xia X, Yang $Y$, Zhou C. Effect of gender on odor identification at different life stages: a meta-analysis. Rhinology. 2019;57(5):322-330.

9. Gane SB, Kelly C, HopkinsC. Rhinology 2020, in press. https://doi.org/10.4193/Rhin20.114.

10. Giacomelli A PL, Conti F, Bernacchia D, Siano $M$ et al. Self-reported olfactory and taste disorders in SARS-CoV-2 patients: a cross-sectional study Clinical Infectious Diseases 2020, https://doi.org/10.1093/cid/ ciaa330.

11. Wang $W, X u Y, G a o$ R, et al. Detection of SARS-CoV-2 in Different Types of Clinical Specimens. JAMA. 2020. doi: 10.1001/ jama.2020.3786.

12. Reden J, Mueller A, Mueller C, et al. Recovery of olfactory function following closed head injury or infections of the upper respiratory tract. Arch Otolaryngol Head Neck Surg. 2006;132(3):265-269.

13. Duncan HJ, Seiden AM. Long-term followup of olfactory loss secondary to head trauma and upper respiratory tract infection. Arch Otolaryngol Head Neck Surg 1995;121(10):1183-1187.

14. Heilmann S, Huettenbrink KB, Hummel T. Local and systemic administration of corticosteroids in the treatment of olfactory loss. Am J Rhinol. 2004;18(1):29-33.

15. https://www.who.int/docs/default-source/ coronaviruse/clinical-management-of-novel-cov.pdf.

16. Hummel T, Heilmann S, Huttenbriuk KB Lipoic acid in the treatment of smell dys- function following viral infection of the upper respiratory tract. Laryngoscope. 2002;112(11):2076-2080.

17. Yan $\mathrm{CH}$, Rathor A, Krook K, et al. Effect of Omega-3 Supplementation in Patients With Smell Dysfunction Following Endoscopic Sellar and Parasellar Tumor Resection: A Multicenter Prospective Randomized Controlled Trial. Neurosurgery. 2020. pii: nyz559. doi: 10.1093/neuros/nyz559.

18. Sorokowska A, Drechsler E, Karwowski M Hummel T. Effects of olfactory training: a meta-analysis. Rhinology. 2017;55(1):17-26.

19. Hummel T, Whitcroft KL, Rueter G, Haehner A. Intranasal vitamin A is beneficial in post-infectious olfactory loss. Eur Arch Otorhinolaryngol. 2017;274(7):2819-2825.

Claire Hopkins

Prof of Rhinology

Guy's Hospital

London SE1 9RT

United Kingdom

Tel: +447971527920

E-mail: clairehopkins@yahoo.com 